\title{
ABUNDANCE PECULIARITIES IN WHITE DWARF ATMOSPHERES AS A RESULT OF BINARY EVOLUTION?
}

\author{
I. Bues \\ Dr.Remeis Sternwarte Bamberg, Astron. Inst.Univ.Erlangen- \\ Niirnberg, G.F.R.
}

We investigate metal abundances in helium-rich white dwarf atmospheres and check the possibility of variations due to binary evolution.

\section{INTRODUCTION}

From the spectroscopically classified sample of white dwarfs, continuously enlarged by Greenstein, it is well known that most of these objects belong to the group of extremely hydrogen-rich DA-stars. If we look for binaries, however, we find only 12 per cent being members of wide binary systems compared to 20 per cent of the whole sample. The two close binary systems - the ultrashort period variables AM CVn and G 6129 - show only helium lines in their spectra.

According to Nather et al.(1979) these systems might be the precursors of very low mass $\left(M<0.4 \mathrm{M}_{\Theta}\right.$ ) single helium-rich DB stars with a helium core since the secondary $\left(M=0.02 \mathrm{M}_{\odot}\right.$ ) is likely to be completely dissolved. At least a certain fraction of the DB-stars and their cooler descendants of spectral type $D C, C_{2}, D F$ and $D G$ should be the result of a process like that. If compared to other stars of the same spectral type, they should have a smaller $\log \mathrm{g}$ and a decreased metal abundance.

Wide binary systems containing a non-DA white dwarf are more massive $\left(M \approx 1 M_{O}\right)$ than the above mentioned systems with a typical separation of $270 \mathrm{AU}$. Both components should have undergone the evolution of single stars with a certain amount of mass loss from the system. We expect degenerate stars with cores of elements heavier than helium (M $0.5 \mathrm{M}_{\odot}$ ) and at least traces of heavier elements in the outer helium-rich atmosphere.

With model atmospheres fitted to observations of helium-rich white dwarfs in the range of $T_{\text {eff }}$ where metal lines occur in the visible and UV spectrum we try to see a difference in mass and/or metal abundances between single stars and binary components.

409

Z. Kopal and J. Rahe (eds.), Binary and Multiple Stars as Tracers of Stellar Evolution, 409-411.

Copyright $\odot 1982$ by D. Reidel Publishing Company. 
II. MODEL ATMOSPHERES AND RESULTS

Flux-constant helium-rich model atmospheres including convection have been computed in the range $12000>\mathrm{T}$ f $>6000 \mathrm{~K}$ with abundance ratio of $10^{6}>\mathrm{He} / \mathrm{H}>10^{3}, 10^{8}>\mathrm{He} / \mathrm{C}>10^{3}, 10^{3}>\mathrm{c} 9 \mathrm{f}^{\mathrm{f}}>1 / 3$, all heavier elements reduced by a factor of $10^{5}$ compared to the solar value. Special emphasis has been taken for the treatment of all molecules, not only their dissociative equilibria but also their absorption of the flux due to strong bands.

(Details of the computations and some model atmospheres will be published in Astronomy \& Astrophysics)

The resulting fluxes have been compared to observed spectra in the visible and UV taken by Wegner $(1973,1980,1981)$, scannerobservations by Oke (1974) and IUE observations by Weidemann et a1. $(1980,1981)$. our results have been compiled in Tables $I$ and II.

TableI : Cool helium-rich white dwarfs (single stars)

\begin{tabular}{lccccccc} 
& $\mathrm{T}_{\text {eff }}$ & log $\mathrm{g}$ & $\mathrm{M} / \mathrm{M}_{\mathrm{O}}$ & $\mathrm{He} / \mathrm{H}$ & $\mathrm{He} / \mathrm{C}$ & $\mathrm{He} / \mathrm{O}$ & $\mathrm{He} / \mathrm{Ca}$ \\
\hline VMa2 & 5800 & 7.42 & 0.31 & $>10^{5}$ & $>10^{7}$ & $>10^{7}$ & $5 \cdot 10^{10}$ \\
R640 & 8300 & 7.5 & 0.45 & $10^{3.6}$ & $>10^{5}$ & $>10^{5}$ & $4 \cdot 10^{8}$ \\
L145-141 & 7500 & 7.8 & 0.40 & $>10^{5}$ & $10^{6}$ & $>10^{8}$ & $>10^{10}$ \\
EG148 & 7500 & 7.5 & & $>10^{5}$ & $5 \cdot 10^{6}$ & $>10^{8}$ & $>10^{10}$ \\
L97-3 & 9800 & 7.5 & & $>10^{5}$ & $2 \cdot 10^{5}$ & $>10^{7}$ & $>10^{7}$ \\
G257-38 & 7000 & 7.5 & & $>10^{5}$ & $5 \cdot 10^{5}$ & $>10^{7}$ & $>10^{10}$ \\
\hline
\end{tabular}

Table II: Cool helium-rich white dwarfs (wide binaries)

\begin{tabular}{llllllll} 
& $\mathrm{T}_{\text {eff }}$ & $\log \mathrm{g}$ & $\mathrm{M} / \mathrm{M}_{0}$ & $\mathrm{He} / \mathrm{H}$ & $\mathrm{He} / \mathrm{C}$ & $\mathrm{He} / \mathrm{O}$ & $\mathrm{He} / \mathrm{Ca}$ \\
\hline BPM27606 & 8200 & 7.8 & 0.82 & $10^{4}$ & $5 \cdot 10^{4}$ & $>5 \cdot 10^{5}$ & $>10^{8}$ \\
G47-18 & 8800 & 7.5 & 0.75 & $10^{5}$ & $3 \cdot 10^{4}$ & $>3 \cdot 10^{5}$ & $>10^{8}$ \\
LDS678B & 10600 & 7.8 & 0.7 & $10^{4}$ & $2 \cdot 10^{4}$ & $>10^{5}$ & $>10^{7}$ \\
G218-8 & 10300 & 7.8 & 0.65 & $>10^{5}$ & $10^{4}$ & $>10^{5}$ & $>10^{7}$ \\
L745-46A & 7500 & 8.0 & 0.8 & $>10^{5}$ & $>10^{8}$ & $>10^{8}$ & $4 \cdot 10^{9}$ \\
\hline
\end{tabular}

For vMa2, the values obtained by Grenfell (1974) have been included. Due to several improvements of the model atmosphere program the results of stars included already in the analysis of Bues (1973) and 
Bues (1979) have been slightly changed. For R640, the effective temperature is lower than earlier work by Liebert (1977) and Cottrel1 and Greenstein (1980).

\section{DISCUSSION}

Although the analyzed sample of cool helium-rich white dwarfs is very small the difference in masses and abundances seems to be significant. If more parallaxes of single white dwarfs could be measured, the mass determination would be improved. Shipman (1979) derived statistical parallaxes from scanner observations of a large sample of white dwarfs.His mass determination of DB stars (his Table 6) compare very well with our result:stars in binary systems have larger masses than single ones.

Concerning the purity of helium in the atmospheres, single degenerates are favoured against binary components independent of spectral type. There is no systematic difference in total metal content between stars with carbon or calcium determined spectra.

So we may conclude that the mechanism proposed by Nather et a1. cannot work for helium-rich binary components but might have been effective for the precursor of vMa2 or L145-141.Cool atmospheres of white dwarfs consisting of helium on $1 y$ have not been observed, the formerly classified DC stars reveal strong features due to metal lines in the far UV region.

\section{REFERENCES}

Bues, I., 1973, Astron. Astrophys. 28, 181

Bues, I., 1979, IAU Co11. 53, Rochester University Press, p. 186

Bues, I., 1981, preprint

Cottre11, P.L., Greenstein, J.L., 1980, Ap. J. 238, 941

Grenfe11, T.C., 1974, Astron. Astrophys. 31, $30 \overline{3}$

Liebert, J.W., 1977, Astron. Astrophys. 60, 101

Nather, R.E., Robinson, E.L., Stover, R.J., 1979, IAU Co11. 53,p. 453

Oke, J.B., 1974, Ap.J. Supp1. 27, 21

Shipman, H.L., 1979, Ap.J. 228, 240

Wegner, G., 1973, Monthly Not. roy.Astron. Soc. 163, 381

Wegner, G., 1981a, Ap.J. 245, L27

Wegner, G., 1981b, Ap.J. $\underline{248}$, L129

Weidemann, V., Koester, D., Vauclair, G., 1980, Astr.Astrophys.83, L13

Weidemann, V., Koester, D., Vauclair, G., 1981, Astr.Astrophys.모, L9 